\title{
Existe diferença na tomada de decisão em situações de levantamento e de bloqueio em atletas escolares de voleibol?
}

Is there a difference in decision-making considering setting and blocking situations in school volleyball athletes?

¿Hay alguna diferencia en la toma de decisiones en las situaciones de colocación y bloqueo de atletas escolares de voleibol?

Rivanildo Constantino dos Santos ${ }^{\mathrm{I}}$, Renata Chavier da Silva ${ }^{\mathrm{II}}$, Samuel da Silva Aguiar ${ }^{\mathrm{III}}$, Gustavo De Conti Teixeira Costa $^{\mathrm{IV}}$, Henrique de Oliveira Castro ${ }^{\mathrm{V}}$

\section{Resumo}

O presente estudo objetiva analisar a qualidade da tomada de decisão (TD) de atletas masculinos escolares de voleibol, comparando as situações de levantamento (LE) e bloqueio (BL). Participaram 15 atletas masculinos escolares de voleibol do Distrito Federal (idade média de 16,6 $\pm 1,1$ anos; tempo de treino sistematizado de 2,8 $\pm 1,6$ anos). Utilizou-se cenas de LE e BL no Teste de Conhecimento Tático Declarativo no Voleibol e o teste t para avaliação da TD. Observou-se uma melhor TD na situação de BL em relação a LE.

Palavras-chave: Cognição; Adolescentes; Tomada de Decisão; Voleibol

\begin{abstract}
This study aims to analyze the quality of decision-making (DM) of male volleyball school athletes, comparing the situations of setting (SE) and blocking (BL). Fifteen male volleyball school athletes from Federal District participated (on the mean of age 16.6 \pm 1.1 years old; systematized training time of $2.8 \pm 1.6$ years). Scenes of SE and BL of Declarative Tactical Knowledge Test in Volleyball were used and the $t$ test was used to evaluate DM. A better DM was observed in the BL situation compared to SE.
\end{abstract}

Keywords: Cognition; Adolescent; Decision Making; Volleyball

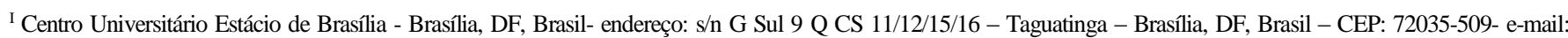
rivatotal@yahoo.com.br

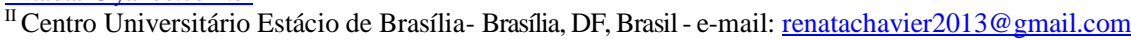

III Centro Universitário do Distrito Federal - UDF- Brasília, DF, Brasil - e-mail: ssaguiar0@ gmail.com

IV Universidade Federal de Goiás - UFG - Goiânia, GO, Brasil - e-mail: conti02@ hotmail.com

v Centro Universitário Instituto de Educação Superior de Brasília - IESB - Brasília, DF, Brasil - e-mail: henriquecastro88@ yahoo.com.br
} 


\section{Resumen}

El estudio tiene como objetivo analizar la calidad de la toma de decisiones (TD) de atletas escolares masculinos de voleibol, comparando las situaciones de colocación (CO) y bloqueo (BL). Participaron quince atletas escolares masculinos de voleibol del Distrito Federal de Brasil (edad media de 16.6 \pm 1.1 años; tiempo de entrenamiento sistematizado de 2.8 \pm 1.6 años). Ha sido utilizada las escenas de CO y BL en el Test de Conocimiento Táctico Declarativo en Voleibol y el test t para evaluar la TD. Se observó una mejor TD en la situación BL en comparación con CO.

Palavras clave: Cognición; Adolescente; Toma de Decisión; Voleibol

\section{Introdução}

O voleibol é uma modalidade esportiva coletiva que se caracteriza por mudanças frequentes nas ações de jogo, as quais solicitam que seus praticantes tomem decisões táticas para solucionar os problemas impostos por determinada situação da partida (GALATTI et al., 2017ab; VILAMALDONADO et al., 2014ab; LIMA; MATIAS; GRECO, 2012; MATIAS; GRECO, 2009; GARGANTA, 2001). Dessa forma, exige-se dos atletas ações tático-técnicas, com muita versatilidade e rapidez nas suas decisões, sendo os processos cognitivos fundamentais para o desempenho (MATIAS; GRECO, 2011).

O comportamento do atleta para a realização da ação é considerado, conforme a literatura, como um comportamento tático, dirigido e regulado psiquicamente, que considera o posicionamento dos colegas e do adversário, o momento do jogo, as características individuais dos atletas, entre outros (PRAÇA et al., 2017; MATIAS, et al., 2016). Assim, a eleição de uma resposta correta em uma situação de jogo (tomada de decisão - TD), bem como a execução motora (técnica), são fatores relevantes no desenvolvimento da expertise nesse tipo de esporte (VILA-MALDONADO et. al., 2014a; DEL VILLAR et al., 2003).

A TD é a capacidade de escolher uma entre diferentes opções em um determinado tempo/momento (MARASSO et al., 2014). A TD pode ser influenciada, dentre outros fatores, pelos números de alternativas e possibilidades de ação para a solução da situação apresentada (MATIAS; GRECO, 2009). De acordo com Greco (2009), é um processo intencional direcionado a um objetivo, considerado como uma "cognição em ação", no qual o atleta solicita da cognição uma resposta para ser realizada a ação.

Diante da grande variabilidade e velocidade do jogo de voleibol, que se solicita TD rápidas e constantes, as ações de levantamento para a finalização da jogada (ataque) e o bloqueio para a defesa com 
ponto direto e/ou possibilidade de contra-ataque, destacam-se como importantes fundamentos do jogo. Portanto, o ritmo do jogo e a qualidade das ações poderão determinar o sucesso do ataque e a probabilidade de pontos e seu respectivo aumento na partida (COSTA et al., 2017).

No jogo de voleibol, o levantador possui um importante significado tático-estratégico ofensivo no jogo, sendo considerado o "cérebro" da equipe (MATIAS; GRECO, 2011). Para tanto, o levantador, dentre todos os demais atletas de uma equipe, tem o objetivo primordial de processar uma maior quantidade e variações de informações durante uma partida, além de tomar as decisões ofensivas do local do levantamento para facilitar o ataque com mais eficácia (MATIAS; GRECO, 2011).

Observa-se também que o bloqueio se caracteriza por uma ação tático-técnica defensiva e ofensiva. Defensiva porque pode ser utilizado para amortecer a potência do ataque da equipe adversária e facilitar o sistema defensivo de sua equipe para um possível contra-ataque. Além disso, o bloqueio pode ser ofensivo, caracterizado pelo objetivo de bloquear o ataque adversário de forma que a bola retorne diretamente para a quadra adversária (CASTRO et al., 2014).

Dessa forma, o levantamento e o bloqueio apresentam-se como importantes ações para o desempenho do atleta no jogo. Observa-se, na literatura específica, a influência da TD no desempenho de atletas de voleibol de diferentes níveis competitivos, porém não foram encontrados estudos que comparassem a TD nas situações específicas de bloqueio e levantamento com atletas em nível escolar. Assim, o presente estudo objetiva analisar a qualidade da tomada de decisão (TD) de atletas masculinos escolares de voleibol, comparando-se as situações de levantamento (LE) e bloqueio (BL).

\section{Métodos}

\section{Amostra}

A amostra foi definida por conveniência (THOMAS; NELSON; SILVERMAN, 2012), de acordo com a disponibilidade e aceitação da escola e dos estudantes convidados à participação no estudo. Foi composta por 15 atletas masculinos escolares de voleibol do Distrito Federal com idade média de

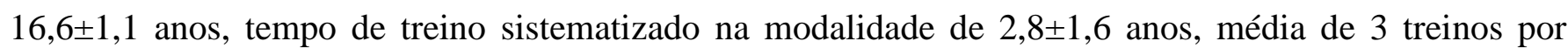
semana com duração de 90 minutos no contra turno escolar, e que disputam competições escolares em nível regional e nacional. Destaca-se que os voluntários também realizam as aulas de Educação Física Escolar além do treinamento de voleibol no contra turno. 


\section{Procedimentos}

O estudo foi aprovado pelo Comitê de Ética em Pesquisas da Universidade (Parecer 971.037) e seguiram todas as orientações éticas propostas pelo Conselho Nacional de Saúde (CNS) para estudos com seres humanos.

As coletas ocorreram na própria escola, em uma sala reservada e propícia para a realização dos procedimentos da pesquisa. Os horários para a aplicação do teste foram previamente agendados com cada voluntário, estando presentes o pesquisador responsável e o voluntário. O teste foi aplicado sempre antes da sessão de treino.

Os voluntários foram conduzidos individualmente até a sala e, primeiramente, preencheram o questionário de dados demográficos, aplicado com o objetivo de caracterizar a amostra e coletar informações importantes para a pesquisa, como utilizado em diversos estudos na referida modalidade (CASTRO et al., 2019, 2017, 2016; COSTA et al., 2016; FERNÁNDEZ-ECHEVERRÍA et al., 2014; GIL et al., 2013; 2011ab).

Em seguida, para avaliar a qualidade da tomada de decisão (TD), os voluntários sentaram confortavelmente em uma cadeira de frente para o computador para análise das cenas de levantamento (LE) e bloqueio (BL) do Teste de Conhecimento Tático Declarativo no Voleibol - TCTD:VB (COSTA et al., 2016). Após as explicações sobre o teste dadas pelo pesquisador, iniciaram-se duas cenas de cada situação para adaptação. Sem nenhuma dúvida ou dificuldade, o voluntário iniciava o teste quando desejasse.

O TCTD:VB consiste em cenas reais de jogos de voleibol apresentadas em dois tipos de situações de jogo: Levantamento - LE (seis cenas), Bloqueio - BL (seis cenas). As cenas exibidas têm duração variável entre quatro a seis segundos, filmadas na perspectiva de topo a uma altura de quatro metros e com distância aproximada de sete a nove metros do fundo da quadra, o que permite ao observador a visão total da quadra e percepção de profundidade nas diferentes situações (COSTA et al., 2016).

As situações de LE iniciam-se a partir do saque adversário, perpassam pela recepção da equipe e, no momento do levantamento, interrompe-se a imagem. Nas situações de BL iniciam-se a partir do saque da sua equipe, perpassa pela recepção da equipe adversária e, no momento do levantamento, interrompese a imagem. No momento da interrupção da cena, a tela se apaga, ficando completamente escura. Nesse momento, o atleta tem que dizer imediatamente "o que fazer" (considera-se essa resposta como sendo a TD). 
Para as situações de LE, as opções esperadas eram: levantamento para a posição 4, levantamento para a posição 3 , levantamento para a posição 2 , levantamento para a posição 1 , levantamento para a posição 6. Para as situações de BL, as opções esperadas eram: bloqueio na posição 2, bloqueio na posição 3, bloqueio na posição 4 .

Esse teste já foi utilizado em outros trabalhos desenvolvidos por membros do Centro de Estudos de Cognição e Ação (CECA/UFMG) com atletas jovens e treinadores de voleibol (CASTRO et al., 2019, 2017, 2016; COSTA et al., 2018).

\section{Análise dos dados}

Foi realizada uma análise descritiva e posteriormente realizou-se o teste t para comparar possíveis diferenças na pontuação final dos testes, número de tomadas de decisão corretas e percentual (\%) de acerto na tomada de decisão entre os fundamentos levantamento e bloqueio. No tratamento dos dados adotou-se o valor de significância de $5 \%(p \leq 0,05)$ e utilizou-se o software SPSS versão 20.0.

\section{Resultados}

A Tabela 1 abaixo apresenta os resultados da análise da comparação da qualidade da TD entre os atletas nas situações de LE e BL.

Tabela 1. TD de atletas masculinos escolares em situações de LE e BL

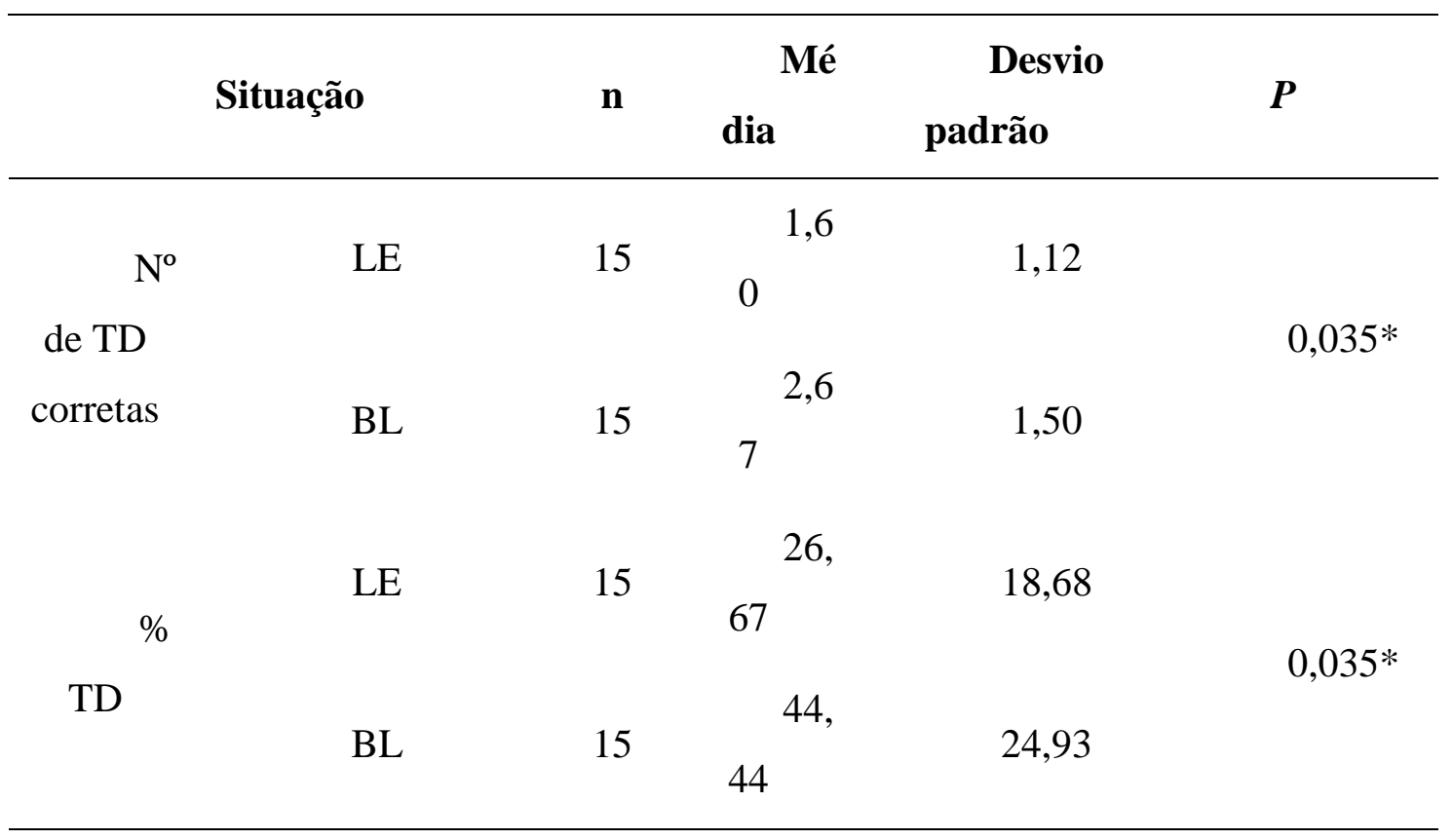




\section{LE=Levantamento; $\mathrm{BL}=$ Bloqueio; $\mathrm{TD}=$ Tomada de Decisão; ${ }^{*} \mathrm{p}<0,05$.}

A análise dos resultados indicou que houve diferenças segundo os fundamentos (Tabela 1). O teste t mostrou que o número de decisões corretas [t(28)=-2,210; $\mathrm{p}=0,035]$ e o \%TD correta $[\mathrm{t}(28)=-2,210$; $\mathrm{p}=0,035]$ foram superiores na análise do fundamento bloqueio em relação ao levantamento.

\section{Discussão}

De acordo com os resultados, atletas escolares masculinos de voleibol tomam melhores decisões nas situações de bloqueio quando comparadas às situações de levantamento. Estudos sugerem que o treinamento tático e técnico sejam realizados de forma situacional, por meio de variados contextos do jogo (WOODS et al., 2019; MESQUITA; PEREIRA; GRAÇA, 2009). Assim, solicita-se dos jogadores uma maior flexibilidade cognitiva e maior capacidade de prever (permitindo-lhes a antecipação das ações), e de adaptar (interpretar), graças às diferentes situações do jogo, favorecendo respostas mais eficazes (WILLIAMS et al., 2009; MCPHERSON, 2008; MESQUITA; GRAÇA, 2002).

A adaptação da TD à situação de jogo configura-se uma questão decisiva em ações de jogo no voleibol, já que a velocidade se constitui em importante "constraint" da ação (AFONSO; MESQUITA, 2011; MESQUITA; CÉSAR, 2007). Além disso, o treinamento da técnica em situações reais de jogo permite o seu aperfeiçoamento em situações variáveis, que é uma exigência da regra da modalidade, visto que o erro da execução da técnica contabiliza ponto para o adversário.

De acordo com Maldonado et al. (2014), atletas mais experientes tomam melhores decisões do que atletas menos experientes, diferença essa definida devido às melhores percepções, a concretização e o recebimento de estímulos relevantes. Estudos como os de Castro et al (2019, 2016, 2013), Costa et al. (2018), Conejero et al. (2017), Moreno et al. (2016) e Afonso et al (2012abc), analisaram a relação entre experiência e a TD de atletas e treinadores através de análises de vídeo ou recursos de feedback. Os resultados demonstraram que atletas com mais experiência no voleibol tomavam melhores decisões nas diferentes situações de jogo.

Araújo, Afonso e Mesquita (2011) demonstraram que atletas com melhores desempenhos competitivos apresentam maiores valores percentuais de decisões corretas quando comparados aos atletas com piores desempenhos competitivos em diferentes categorias do voleibol. Resultados semelhantes foram encontrados por Castro et al. (2016) na comparação entre atletas de voleibol e de outras 
modalidades utilizando-se do TCTD:VB. No presente estudo, não há comparação da percepção de atletas mais ou menos experientes e do desempenho competitivo dos atletas, somente a TD dos atletas escolares em duas diferentes situações de jogo.

Apesar de não haver a comparação das variáveis supracitadas, os resultados do presente estudo sugerem que os sinais relevantes percebidos e analisados na ação de bloqueio são diferentes da situação de levantamento, sendo que situações de bloqueio detêm menores possibilidades de ação do que as situações de levantamento, o que pode ser um fator que explica a diferença encontrada na comparação dos atletas escolares.

Castro et al. (2014) analisaram a interação das ações de saque e bloqueio e demonstraram que a ação de bloqueio fica condicionada a outras ações como saque e sistema defensivo/recepção. Observa-se que a TD no bloqueio ficou mais evidente pela atenção dada ao saque e recepção, levando os escolares a melhores resultados no bloqueio, o que corrobora com os resultados do presente estudo.

Moura, Monteiro e Batista (2018) realizaram uma intervenção de 25 aulas com estudantes entre 14 e 17 anos de idade, utilizando o Step-Game-Approach e o Teaching Games for Understanding (TGfU) na aprendizagem do voleibol. Os resultados desse estudo apontam melhoras significativas na TD dos participantes, o que corrobora com os achados no presente estudo para a situação de bloqueio. De acordo com os autores, a TD deve ser trabalhada através do direcionamento da atenção com o objetivo de melhoria do conhecimento tático e reconhecimento dos sinais relevantes, o que leva a melhora da TD propriamente dita.

\section{Conclusões}

De acordo com os resultados apresentados, conclui-se que atletas escolares masculinos de voleibol possuem melhor tomada de decisão nas situações de bloqueio quando comparados as situações de levantamento.

Do ponto de vista prático, os treinadores devem enfatizar no treinamento situações para o desenvolvimento da leitura de jogo, antecipação, interpretação e tomada de decisão. Dessa forma, pode-se utilizar propostas para o ensino-aprendizado-treinamento já descritos na literatura especializada como a Iniciação Esportiva Universal - IEU (GRECO, 2001, 1998; GRECO; BENDA, 1998), a Escola da Bola EB (ROTH; KROGER; MEMMERT, 2017; KROGER; ROTH, 2002), o Sport Education Model - SEM 
(SIEDENTOP, 1994), o Teaching Games for Understanding - TGfU (BUNKER; THORPE, 1982), bastante utilizados para a iniciação esportiva de jogos coletivos.

Além disso, de acordo com os resultados encontrados no presente estudo, os treinadores desse público específico devem priorizar o treinamento de situações de levantamento no sentido de melhorar a percepção, a leitura do jogo e, por consequência, a tomada de decisão desses atletas.

\section{Referências}

AFONSO, J.; ESTEVES, F.; ARAÚJO, R.; THOMAS, L.; MESQUITA, I. Tactical determinants of setting zone in elite men's volleyball. Journal of Sports Science and Medicine, v.11, p.64-70, 2012a.

AFONSO, J.; GARGANTA, J.; MCROBERT, A.; WILLIAMS, A.M.; MESQUITA, I. The perceptual cognitive processes underpinning skilled performance in volleyball: Evidence from eye-movements and verbal reports of thinking involving an in situ representative task. Journal of Sports Science and Medicine, v.11, p.339-345, 2012b.

AFONSO, J.; GARGANTA, J.; MCROBERT, A.; WILLIAMS, M.; MESQUITA, I. Visual search behaviors and verbal reports during film-based and in situ representative tasks in volleyball. European Journal of Sports Science, v.14, n.2, p.1-8, 2012c.

AFONSO, J.; MESQUITA, I. Determinants of block cohesiveness and attack efficacy in high level women's volleyball. European Journal of Sport Science, v.11, n.1, p.69-75, 2011.

ARAÚJO, R.; AFONSO, J.; MESQUITA, I. Procedural knowledge, decision-making and game performance analysis in Female Volleyball's attack according to the player's experience and competitive success. International Journal of Performance Analysis in Sport, v.11, p.1-13, 2011

BUNKER, D.; THORPE, R. A model for the teaching of games in secondary schools. Bulletin of Physical Education, v.18, p.5-8, 1982.

CASTRO, H. O.; COSTA, G. C. T.; PRAÇA, G. M.; CAMPBELL, C. S. G.; GRECO, P. J. Análise das fixações visuais e da tomada de decisão de atletas de voleibol das m categorias infanto e juvenil. Revista Brasileira de Ciência e Movimento, v. 25, n. 1, p. 51-59, 2017.

CASTRO, H. O.; COSTA, G. C. T.; PRAÇA, G. M.; LAGE, G.M; FERNADEZ-ENCHEVERRIA, C; GRECO, P. J. Visual behaviour and decision-making in attack situations in volleyball. Revista Internacional de Medicina y Ciencias de la Actividad Fisica y Deporte, v. 19, n.75, p. 565-578, 2019.

CASTRO, H. O.; COSTA, G. C. T.; PRAÇA, G. M.; PEDROSA, G. F.; GRECO, P. J. Visual behaviour and the quality of decision-making on volleyball. Revista Brasileira de Cineantropometria $\mathrm{e}$ Desempenho Humano, v. 18, n. 6, p. 638-647, 2016. 
CASTRO, H. O.; CAVALLI, I.; SILVA, C. J. A.; GRECO, P. J. Interação no curso das ações de saque e bloqueio no voleibol juvenil. Conexões, v. 12, n. 3, p. 34-54, 2014.

CASTRO, H.O.; ZACHARIAS, G.F.R.; MATIAS, C.J.A.S.; GRECO, P.J. Conhecimento tático declarativo dos levantadores da seleção brasileira juvenil masculina de Voleibol. Revista Mineira de Educação Física, Edição Especial, n.9, p.1069-1073, 2013.

CONEJERO, M.; CLAVER, F.; FERNÁNDEZ-ECHEVERRÍA, C.; MORENO, M.P. Relationship between decision-making and performance in game actions in volleyball. European Journal of Human Movement, v.39, p.82-95, 2017.

COSTA G.C. O.; CASTRO, H. O.; EVANGELISTA, B. F. I.; LAURA, M. M.; GRECO, P. J. Predinting factors of zone 4 attack in volleyball. Peceptual and Motor Skills, v. 0, n. 0, p. 1-13, 2017.

COSTA, G.C.T.; CASTRO, H.O.; MESQUITA, I.M.R.; AFONSO, J.; LAGE, G.M.; GRECO, P.J. Visual Search and Decision-Making of Volleyball Coaches. Journal of Physical Education, v.29, e2934, 2018.

COSTA G.C. O.; CASTRO, H. O.; CABRAL, F. A., MORALES, J. C.; GRECO, P. J. Content Validity of scenes of the eclarative Taical Knowlwdge Test in volleyball-DTKT. Revista Brasileira de Cineantropometria e Desempenho Humano, v. 18, n. 6, p. 629-637, 2016.

DEL VILlAR, F.; IGLESIAS, D.; MORENO, M. P.; FUENTES, J. P.; CERVELlÓ, E. M. An investigation into procedural knowledge and decision-making: Spanish experiencer-inexperienced basketball players differences. Journal of Human Movement Studies, v. 46, p. 407-420, 2003.

FERnÁNDEZ- ECHEVERriA, C.; ARROYO, A. M.; ARIAS, A. G. RABAZ, F. C.; ARROYO, M. P. M. Estudio del conocimento procedimental experiência y rendimento em jóvenes jugadores de voleibol. Retos, v. 25, p. 13-16, 2014.

GALATTI, L.R.; BETTEGA, O.B.; PAES, R.R.; REVERDITO, R.S.; SEOANE, A.M.; SCAGLIA, A.J. O ensino dos jogos esportivos coletivos: avanços metodológicos dos aspectos estratégico-tático-técnicos. Pensar a Prática, v.20, n.3, p.639-654, 2017a.

GALATTI, L.R. et al. (Org.) Desenvolvimento de treinadores e atletas. Pedagogia do Esporte. v.1, Campinas/SP: Editora Unicamp, 2017 b.

GARGANTA, J. A análise da performance nos jogos desportivos: Revisão acerca da análise do jogo. Revista Portuguesa de Ciências do Desporto, v. 1, n. 1, p. 57-64, 2001.

GIL, A.; DEL VILLAR, F.; MORENO, A.; GARCIA- GONZÃLEZ, L.; MORENO, M. P. El . Estudio conocimento declarative yprocediemntal em voleibol? Es determinte la categoria de juego para su desarrollo? Motricidad. European Journal of Human Movement, v. 27, p. 117-130, 2011 a.

GIL, A.; MORENO, M. P.; MORENO, A.; GARCÍA-GONZÁLEZ, L.; CLAVER, F.; DEL VILLAR, F. Analysis of the Relationship Between the Amount of Training and Cognitive Expertise. A Study of Young Volleyball Players. Journal of Strength \& Conditioning Research, v.27, n.3, p.698-702, 2013. 
GIL, A.; MORENO, M. P.; MORENO, A.; GARCÍA-GONZÁLEZ, L.; DEL VILLAR, F. La práctica federada como elemento de desarrollo del conocimiento: Aplicación al voleibol de formación. International Journal of Sport Science, v.7, n.24, p.230-245, 2011 b.

GRECO, P.J. Tomada de decisão no esporte. In.: SAMULSKI, D.M. (Ed). Psicologia do Esporte: conceitos e novas perspectivas. $2^{\mathrm{a}}$ ed., Barueri/SP: Editora Manole, 2009, p.107-142.

GRECO, P.J. Iniciação Esportiva Universal: metodologia da iniciação esportiva na escola e no clube. v.2. Belo Horizonte: Editora UFMG, 1998.

GRECO, P.J. Métodos de ensino-aprendizagem-treinamento nos jogos esportivos coletivos. In. GARCIA, E.S.; MOREIRA, K.L. Temas Atuais IV. Belo Horizonte: Editora Health. 2001. p. 48-72.

GRECO, J.P.; BENDA, R.N. Iniciação Esportiva Universal. Da aprendizagem motora ao treinamento técnico. v.1, Belo Horizonte: Editora UFMG, 1998.

KROGER, C.; ROTH, K. Escola da Bola: um ABC para iniciantes nos jogos esportivos. São Paulo: Editora Phorte, 2002.

LIMA, C. O. V.; MATIAS, C. J. A. S.; GRECO, P. J. O conhecimento tático produto de métodos de ensino combinados e aplicados em sequências inversas no voleibol. Revista Brasileira de Educação Física e Esporte, v. 26, n. 1, p. 129-147, 2012.

MARASSO, D.; LABORDE, S.; BARDAGLIO, G.; RAAB, M. A developmental perspective on decision-making in sports. International Review of Sport and Exercise Psychlogy, v.7, n.1, p.251-273, 2014.

MATIAS, C. J. A. S.; GRECO, P. J. Conhecimento tático-estratégico dos levantadores brasileiros campeões de voleibol: da formação ao alto nível. Revista Brasileira de Educação Física e Esporte, v.25, n.3, p.513-535, 2011.

MATIAS, C. J. A. S; GRECO, P. J. Análise de jogo nos jogos esportivos coletivos: a exemplo do voleibol. Pensar a Prática, v.12, n.3, p.1-16, 2009.

MATIAS, S. J. A. S.; FREIRE, A. B.; CASTRO, H. O.; COSTA, G. C. T. Conhecimento tático declarativo no voleibol: diferença entre atletas da categoria sub-15 e sub-17. ALESDE, v. 7, n. 1, p. 39$50,2016$.

MCPHERSON, S.L. Tactics: Using knowledge to enhance performance. In FARROW, D.; BAKER, J.; MACMAHON, C. (Eds.) Developing sport expertise: researchers and coaches put theory into practice. London/England: Routledge, 2008. p.155-167.

MESQUITA, I.; CÉSAR, B. Characterisation of the opposite player's attack from the opposition block characteristics. An applied study in the Athenas Olympic games in female volleyball teams. International Journal Performance Analysis in Sport, v.7, n.2, p.13-27, 2007.

MESQUITA, I.; GRAÇA, A. Probing the strategic knowledge of an elite volleyball setter: A case study. International Journal of Volleyball Research, v.5, n.1, p.6-12, 2002. 
MESQUITA, I.M.R.; PEREIRA, F.R.M.; GRAÇA, A.B.S. Modelos de ensino dos jogos desportivos: investigação e ilações para a prática. Motriz, v.15, n.4, p.944-954, 2009.

MORENO, M.P.M.; MORENO, A.; GARCÍA-GONZÁLEZ, L.; UREÑA, A.; HERNÁNDEZ, C.; DEL VILLAR, F. An intervention based on vídeo feedback and questioning to improve tactical knowledge in expert female volleyball players. Perceptual and Motor Skills, v.122, n.3, p.911-932, 2016.

MOURA, A.; MONTEIRO, J.; BATISTA, P. Impacto da implementação de modelos centrados no aluno na aprendizagem do voleibol. Journal of Sport Pedagogy and Research, v.4, n.2, p.39-50, 2018.

PRAÇA, G.M.; SOUSA, R.B.; SILVA, J.V.O.; CONSTANTINO, F.G.; MOREIRA, P.E.D.; CUSTÓDIO, I.J.O.; MORALES, J.C.P.; GRECO, P.J. Tactical behavior of U-15 soccer players: assessment of changes over a season. Revista Brasileira de Cineantropometria e Desempenho Humano, v.19, n.2, p.251-9, 2017.

ROTH, K.; KROGER, C.; MEMMERT, D. Escola da Bola: Jogos de Rede e Raquete. São Paulo: Editora Phorte, 2017.

SIEDENTOP, D. Sport education: quality pe through positive sport experiences. Champaign, IL: Human Kinetics, 1994.

THOMAS, J.R; NELSON, J.K.; SILVEMAN, S.J. Modelos de Pesquisa em Atividade Física. $6^{\text {a }}$ ed., São Paulo: Editora Artmed, 2012.

VILA-MALDONADO，S.; ABELLÁN， J.; SAÉZ-GALLEGO, N. M.; GARCÍA-LÓPEZ, L. M.; CONTRERAS, O. R. Decision-making and visual perception skills in youth volleyball players and nonplayers. Journal of Sport and Health Research, v. 6, n. 3, p. 265-276, 2014a.

VILA-MALDONADO, S.; SAÉZ-GALLEGO, N. M.; ABELLÁN, J.; GARCÍA-LÓPEZ, L. M.; Analisis de La toma de decisiones em La acción de bloque em voleibol:Comaaración entre jugadoras de elite y amateur. Revista de Psicología del Desporte, v. 23, n. 2, p. 239-246, 2014 b.

WILLIAMS, A.M.; HUYS, R.; CAÑAL-BRULAND, R.; HAGEMANN, N. The dynamical information underpinning anticipation skill. Human Movement Science, v.28, p.362-70, 2009.

WOODS, C.T.; MCKEOWN, I.; SHUTTLEWORTH, R.J.; DAVIDS, K.; ROBERTSON, S. Training programme designs in professional team sport: An ecological dynamics exemplar. Human Movement Science, v.66, p.318-26, 2019.

\section{Como citar este artigo}

SANTOS, R. C.; SILVA, R. C.; AGUIAR, S. S.; COSTA, G. C. T.; CASTRO, H. O. EXISTE Diferença na tomada de decisão em situações de levantamento e de bloqueio em atletas escolares de voleibol? Revista Kinesis, Santa Maria, v. 38, p.01-11, 2020. 